\title{
Image-Matching Digital Macro-Slide-A Novel Pathological Examination Method For Microvascular Invasion Detection In Hepatocellular Carcinoma
}

\author{
Hong-Ming Yu \\ Second Military Medical University \\ Kang Wang \\ Second Military Medical University \\ Jin-Kai Feng
}

Second Military Medical University

Lei Lu

Second Military Medical University

Yu-Chen Qin

Second Military Medical University

Yu-Qiang Cheng

Second Military Medical University

Wei-Xing Guo

Second Military Medical University

Jie Shi

Second Military Medical University

Wen-Ming Cong

Second Military Medical University

Wan Yee Lau

The Chinese University of Hong Kong

Hui Dong

Second Military Medical University

Shu-Qun Cheng ( $\nabla$ chengshuqun@aliyun.com )

Second Military Medical University https://orcid.org/0000-0001-6760-7470

\section{Research Article}

Keywords: Hepatocellular carcinoma, Microvascular invasion, 3-Point baseline sampling protocol, 7-Point baseline sampling protocol, Imaging-matching digital macro-slide, recurrence-free survival, Receiver 
operating characteristic curve, AFP, PIVKA-II

Posted Date: October 27th, 2021

DOI: https://doi.org/10.21203/rs.3.rs-1006518/v1

License: (c) (1) This work is licensed under a Creative Commons Attribution 4.0 International License. Read Full License

Version of Record: A version of this preprint was published at Hepatology International on March 16th, 2022. See the published version at https://doi.org/10.1007/s12072-022-10307-w. 


\section{Abstract}

Background: Microvascular invasion (MVI) is a prominent risk factor of postoperative recurrence for hepatocellular carcinoma (HCC). The MVI detection rate of conventional pathological examination approaches is relatively low and unsatisfactory.

Methods: By integrating pathological macro-slide with whole-mount slide imaging, we first created a novel pathological examination method called image-matching digital macro-slide (IDS). Surgical samples from eligible patients were collected to make IDS. The MVI detection rates, tumor recurrence rates and recurrence-free survival were compared among conventional 3-Point and 7-Point baseline sampling protocols and IDS. Additionally, biomarkers to recognize MVI false negative patients were probed via combining conventional pathological sampling protocols and IDS. Receiver operating characteristic curve (ROC) analysis was used to obtain the optimal cutoff of biomarkers to distinguish MVI false negative status.

Results: The MVI detection rates were $21.98 \%, 32.97 \%$ and $63.74 \%$, respectively, in 3-Point, 7-Point baseline sampling protocols and IDS $(P<0.001)$. Tumor recurrence rate of patients with MVI negative status in IDS (6.06\%) was relatively lower than that of patients with MVI negative status in 3-Point (16.90\%) and 7-Point (16.39\%) sampling protocols. Alpha-fetoprotein (AFP) and protein induced by vitamin $\mathrm{K}$ absence or antagonist-II (PIVKA-II) was selected as potential biomarkers to distinguish MVI false negative patients.

Conclusions: Our study demonstrated that IDS can help enhance the detection rate of MVI in HCC and refine the prediction of HCC prognosis. Alpha-fetoprotein is identified as a suitable and robust biomarker to recognize MVI false-negative patients in conventional pathological protocols.

\section{Introduction}

Hepatocellular carcinoma (HCC) is the fourth leading cause of cancer-related mortality worldwide and the leading cause of death among patients with cirrhosis (1). HCC is characterized by an aggressive clinical course and dismal outcomes, with about two thirds of patients diagnosed at advanced stage at first presentation. Up to $70-80 \%$ of HCC patients will develop disease recurrence within five years following initial curative treatments (1).

Microvascular invasion ( $\mathrm{MVI}$ ) refers to the presence of tumor cell clusters in a vascular lumen lined by endothelial cells under microscopic examination $(2,3)$. It has been repeatedly demonstrated to be a prominent risk factors of postoperative recurrence for $\operatorname{HCC}$ patient $(4,5)$. It is well known that pathological examination is the gold standard to diagnose and grade cancers via observing the morphological character, differentiation degree and growth pattern of tumor cells. Besides, pathological examination can detect some important biological factors around tumor tissues, including microsatellites and MVI. Nevertheless, due to limited scope of routine glass slides and randomness and bias of 
sampling, conventional pathological testing protocol has a tendency to under-report the incidence of MVI in HCC. Hence, constructing a novel pathological technique with greater MVI detection power is required.

Recently, based upon development of histological slide digitization and computational image processing, whole-mount slides imaging (WSI) has been popularized in oncology studies. WSI refers to scanning a complete microscope slide, capturing many small high-resolution image tiles or strips and then montaging them to create a full image of a histopathological section. Many studies have highlighted the clinical significance of WSI in assisting pathologists investigating the whole spectrum of tumor biopsy specimens, and in identifying prognostic markers and histological subtypes of various cancers (6-8). Macroscopic histological slide (macro-slide), different from routine small slides, expands to cover the whole section of tumor tissues and maintains the integrity of tumor specimens to the greatest extent. Macro-slide has the advantage to exhibit more pathological features compared with conventional small slides.

In this study, by combining macro-slide and WSI technique, we create and first report the clinical utility of a novel pathological examination method called "image-matching digital macro-slide (IDS)" for MVI detection in HCC. We show that IDS has the capacity to remarkably increase MVI detection rates in HCC, guiding postoperative adjuvant therapies and surveillance protocols, thus reducing long-term recurrence rates. By analyzing IDS, we are able to get deeper insight into the comprehensive and most relevant features of tumors.

\section{Methods And Materials}

\section{Patients}

Consecutive patients who underwent radical liver resection of HCC at Eastern Hepatobiliary Surgery Hospital (EHBH) from October 2018 to December 2019 were enrolled. The follow-up date was censored on January 31,2021 . The inclusion criteria were as follows: (I) patients aged from 20 to 70 years old, (II) Child-Pugh class A-B7, (III) Eastern Cooperative Oncology Group (ECOG) performance score was 0-1, and (IV) underwent radical resection and had complete postoperative histopathological tissues. The exclusion criteria were as follows: (I) palliative-intend resection, (II) presence of extrahepatic metastasis or major vascular invasion, (III) underwent preoperative anti-cancer treatment, and (IV) a history of other malignant tumors. The study was approved by institutional review board of our hospital. Written informed consent was obtained from patients for their data to be used for research purposes.

\section{Pathological diagnosis}

Two pathologists identified HCC and MVI in all cases, and a third pathologist participated in the identification and gave the final result when there was ambiguity. MVI was defined as a cancer cell cluster composed of $\geq 50$ cells in a microscopic vessel adjacent to the primary tumor.

\section{Materials}


The following materials were required in this study: (I) pathological sampling console, wax block embedding machine, slicer, cooler, dehydrator, slice drying machine, and high-resolution slice scanner; (II) Tissue embedding box $(7.7 \mathrm{~cm} \times 4.3 \mathrm{~cm} \times 1.3 \mathrm{~cm})$, custom-made anti-off slide glass (7.5 $\mathrm{cm} \times 5.0 \mathrm{~cm}$ ), and custom-made cover glass $(6.0 \mathrm{~cm} \times 5.0 \mathrm{~cm})$; (III) special paraffin embedding mold, large wax block holder, and special rack; (IV) Olympus Automatic Digital Pathology Scanner (VS120).

\section{Intraoperative marking}

When intraoperative exploration, we determined the surgical resection range and marked the cutting edge and direction. It was recommended that the cross-section of the human body be taken as the sampling plane, marked with two asymmetric directions, and photographed for filing. Hence, it was more convenient to distinguish the direction of the tumor specimen in vitro.

\section{Specimen processing}

We determined the section according to the maximum diameter of the tumor, half of which were taken according to the 3-Point and 7-Point baseline sampling protocols (Fig. 1A; Fig. 1B), the other half of which were taken using the IDS method (Fig. 1C). After isolation, $10 \%$ neutral formaldehyde should be injected as soon as possible (within $30 \mathrm{~min}$ ) and fixed continuously for no less than $48 \mathrm{~h}$. According to the direction of sampling and section, the thickness of the specimen was $0.5-1.0 \mathrm{~cm}$, and the maximum size of the trimmed specimen was $6.0 \mathrm{~cm} \times 5.0 \mathrm{~cm}$. If the specimen was too large, it could be divided into several parts as required. Then, paraffin embedding, serial slicing and Hematoxylin-Eosin (HE) staining were carried out in sequence.

\section{Digital scanning and analysis}

All tissue sections were scanned with a high-resolution slice scanner and corresponding image data were stored. The images were analyzed using the software matched with the scanner for parameters such as micro-metastases, stromal cell proportion, peripheral inflammatory changes, etc. The image data were matched with the scanned images, and the characteristics of pathological and imaging changes were analyzed again. Digital image signals in the region could be analyzed according to the research content.

\section{Follow-up}

Patients were followed up with laboratory tests including tumor biomarkers and liver biochemistry, abdominal ultrasonography, and contrast-enhanced CT once every 3-6 months. The diagnosis of intrahepatic recurrence was made by imaging findings alone if the tumor displayed typical enhancement characteristics; otherwise, the recurrent diseases were biopsied. Recurrence-free survival (RFS) time was calculated from the date of first surgery to the date when there was a clear evidence of recurrent disease.

\section{Statistical analysis}


Continuous variables were expressed as mean (standard deviation) or median (interquartile range) and compared using student's $t$ test or Mann-Whitney $U$ test according to the distribution of variables. Categorical variables were compared using chi-square test or Fisher's exact test. Survival curves were generated using the Kaplan-Meier method and compared using the log-rank test. Receiver operating characteristic curve (ROC) analysis was used to obtain the optimal cutoff value of AFP and PIVKA-II to distinguish MVI false negative patients. Clinical performance of 3-Piont, 7-Point and IDS to identify MVI actual positive was assessed by the sensitivity, specificity, and predictive values. Statistical analyses were performed by SPSS 26.0 software (SPSS, Inc., Chicago, IL, USA) and R 3.6.3 software (R Development Core Team). $\mathrm{P}$ values less than 0.05 indicated statistical significance.

\section{Results}

\section{The detailed process of IDS}

Preoperative MRI showed that the tumor was located in the posterior lower segment of the right lobe of the liver. The size of the tumor was $72 \mathrm{~mm} \star 58 \mathrm{~mm} \star 55 \mathrm{~mm}$. T2W1 showed slightly higher, equal, and uneven equal-high signals. Scanning after enhancement, the hepatic artery phase demonstrated obvious uneven enhancement. In the portal phase and delayed phase, the relative signal attenuation was in line with the primary liver cancer (Fig. 2A). The preoperative evaluation was in accordance with the inclusion criteria. During the operation, the tumor was completely resected (R0 resection), and the whole specimen was taken to produce macro-slide. After hematoxylin-eosin staining, the macro-slide was observed at different positions with various magnifications (Fig. 2B). Then, the pathological macro-slide was matched with tumor specimen and imaging data to obtain WSI including MVI positions (Fig. 2C). This total process was named as IDS.

\section{The baseline characteristics and long-term outcomes of eligible patients}

A total of 110 primary liver cancer patients were collected in this study. 19 patients who were pathologically diagnosed as intrahepatic cholangiocarcinoma were excluded, and $91 \mathrm{HCC}$ patients were finally included. The baseline clinicopathological characteristics were shown in Table 1. Almost all patients had hepatitis B or $\mathrm{C}$ virus infection background. The percentage of hepatitis $B$ virus infection was $96.7 \%$, and only one patient did not had hepatitis. The liver function of patients was all graded as Child-Pugh class A. The median tumor size was $3.80 \mathrm{~cm}$. In $4 \mathrm{HCC}$ patients associated with portal vein tumor thrombus (PVTT), PVTT existed in the branches of main portal vein and could be resected radically. The shortest and longest follow-up times were 13 and 28 months, respectively. 24 (26.37\%) patients developed disease recurrence and 5 patients (5.49\%) died during follow-up.

\section{MVI detection rates in 3-Point, 7-Point baseline sampling protocols and IDS}

As shown in Fig. 3A, the detection rates of MVI were $21.98 \%, 32.97 \%$ and $63.74 \%$, respectively, in 3-Point, 7-Point and IDS $(P<0.001)$. Patients with MVI positive status in 3-Point and 7-Point were all included in MVI positive status in IDS. The populations of 3-Point and 7-Point were not exactly the same (Fig. 3B). 
Among patients with MVI negative status in 3-Point and 7-Point, the two populations were partly different, but they all included MVI negative status in IDS (Fig. 3C). Therefore, in this study, the specificity and sensitivity of IDS on MVI detection were both $100 \%$, while the specificity of 3-Point and 7-Point on MVI detection was both $100 \%$, and the sensitivity of 3-Point and 7-Point on MVI detection was only $34 \%$ and $52 \%$, respectively (Table 2 ). The above results showed that IDS had superior sensitivity and specificity for the detection of MVI than 3-Point and 7-Point.

\section{Tumor recurrence rates in three various pathological examination methods}

To compare the impact of MVI, which was detected by 3-Point, 7-Point and IDS, respectively, on tumor recurrence, survival analyses were performed (Fig. 4A-C). Under the three methods, patients with MVI positive status were more likely to relapse $(P<0.001, P<0.001, P=0.001$, respectively). We found that 12 (16.90\%) HCC patients with MVI negative status in 3-Point had tumor recurrence, and 2 patients died due to disease progression. In 7-Point sampling method, 10 (16.39\%) patients with MVI negative status had recurrence (relapse time range: $2.1-14.4$ months), including 2 recurrence-related deaths. In IDS, 2 (6.06\%) patients with MVI negative status recurred (relapse time: 13.13 and 14.40 months), and no deaths occurred. Next, we did subgroup analysis combining IDS with 3-Point and 7-Point. Results showed that patients with MVI positive status in both 3-Point and IDS, and in both 7-Point and IDS were most prone to recur. Patients with MVI positive status in IDS were more likely to relapse than patients with actual MVI negative status $(P=0.021, P=0.016)$. (Fig. 4D-E). It is to say, 3-Point and 7-Point sampling protocols have a potential possibility to miss MVI, and patients with MVI false negative status in 3-Point and 7Point are more likely to recur than patients with actual MVI negative status in all three MVI pathological testing methods.

\section{Identification of potential biomarkers to distinguish MVI false negative patients in conventional pathological sampling protocols}

In order to find out the clinicopathological characteristics of patients with missed MVI in 3-Point and 7Point, IDS was matched with 3-Point and 7-Point. As shown in Table 3, There were significant differences in 3-Point in AFP, PIVKA-II, ALP, and tumor number. The medians of AFP between 3-Point negative IDS negative and 3-Point negative IDS positive groups were $6.10(3.10,20.30) \mathrm{ug} / \mathrm{L}$ and $81.30(10.12,560.65)$ $\mathrm{ug} / \mathrm{L}$. The medians of PIVKAll between 3-Point negative IDS negative and 3-Point negative IDS positive groups were $107.00(33.00,412.00) \mathrm{mAU} / \mathrm{mL}$ and $449.00(90.50,3168.75) \mathrm{mAU} / \mathrm{mL}$. The medians of ALP between 3-Point negative IDS negative and 3-Point negative IDS positive groups were 79.00 (63.00, 92.00) U/L and $64.50(54.25,79.25) \mathrm{U} / \mathrm{L}$. As shown in Table 4, only AFP and PIVKA-II had significant differences in 7-Point. The medians of AFP between 7-Point negative IDS negative and 7-Point negative IDS positive groups were $6.10(3.10,20.30) \mathrm{ug} / \mathrm{L}$ and $160.55(20.92,1210.00) \mathrm{ug} / \mathrm{L}$. The medians of PIVKA-II between 7-Point negative IDS negative and 7-Point negative IDS positive groups were 107.00 $(33.00,412.00) \mathrm{mAU} / \mathrm{mL}$ and $460.50(239.25,3084.25) \mathrm{mAU} / \mathrm{mL}$, respectively. It revealed that AFP and PIVKA-Il could be potential biomarkers to distinguish MVI false negative patients in 3-Point and 7-Point. 
In order to study the sensitivity and specificity of AFP and PIVKA-II to identify MVI false negative patients, 71 patients with MVI positive and negative status in IDS but both negative in 3-Point were compared, and a total of 61 patients with MVI positive and negative status in IDS but both negative in 7-Point were compared. After calculating the best cutoff by maximizing the Youden index (Table 5), in 3-Point, when AFP was divided by a cutoff of $22.5 \mathrm{ng} / \mathrm{mL}$, the AUC was $0.715(0.592-0.837)$, the sensitivity was 0.68 (0.51-0.82), and the specificity was $0.79(0.61-0.91)$. When using $267 \mathrm{mAU} / \mathrm{mL}$ as the cutoff of PIVKA-II, the AUC was $0.665(0.538-0.793)$, the sensitivity was $0.66(0.49-0.80)$, and the specificity was 0.67 (0.48-0.82) (Fig. 5A-B). In 7-Point, when AFP was divided by a cutoff of $23.9 \mathrm{ng} / \mathrm{mL}$, the AUC was 0.748 (0.617-0.879), the sensitivity was $0.75(0.55-0.89)$, and the specificity was $0.79(0.61-0.91)$. When using $267 \mathrm{mAU} / \mathrm{mL}$ as the cutoff of PIVKA-II, the AUC was 0.696 (0.558-0.833), the sensitivity was 0.75 (0.55-0.89), and the specificity was 0.67 (0.47-0.81) (Fig. 5C-D). Therefore, AFP is superior to PIVKA-II as a biomarker to distinguish MVI false negative patients.

\section{Potential clinical utility of upper limit of AFP normal value to identify MVI false negative patients in conventional pathological sampling protocols}

Because the cutoff values of AFP in 3-Point and 7-Point (22.5 and $23.9 \mathrm{ng} / \mathrm{mL}$ ) were similar to the upper limit of normal value of AFP $(20 \mathrm{ng} / \mathrm{mL})$, the comparison and analysis were carried out using $22.5 \mathrm{ng} / \mathrm{mL}$ and $20 \mathrm{ng} / \mathrm{mL}, 23.9 \mathrm{ng} / \mathrm{mL}$ and $20 \mathrm{ng} / \mathrm{mL}$ respectively. In 3-Point sampling method, the detection rate of patients with MVI false negative status was $68.4 \%$ (26/38), and there was a significant difference (Fig. $5 \mathrm{E}$, Table 6). In 7-Point sampling method (Fig. 5F, Table 7), the detection rates of patients with MVI false negative status patients were $75 \%(21 / 28)$ and $70 \%(21 / 30)$ respectively, both of which were significantly different. That is to say, in 3-Point and 7-Point, $68.4 \%$ and $70 \%$ of patients with MVI negative status and with AFP greater than $20 \mathrm{ng} / \mathrm{mL}$ are likely to be false negative.

\section{Discussion}

$\mathrm{HCC}$ is characterized as a complex and heterogeneous disease with diverse individual outcomes. MVI is one of the most prominent features and important prognostic factors of long-term survival in HCC. The reported detection rate of $\mathrm{MVI}$ in $\mathrm{HCC}$ was relatively low in previous studies, ranging from $15.0 \%$ to $33.8 \%$ after liver resection or transplantation (9-12). Because of the limited range of ordinary glass slices and the varied criteria in pathological sampling protocol, the conventional pathological testing approach tends to undervalue the presence of MVI in HCC. Therefore, establishing a novel and practical pathological examination approach which can increase MVI detection rate is urgently needed.

With the breakthrough of WSI techniques, high-resolution macro-pathological digital pictures can be acquired. After combining the digital pathological macro-slide data with the imaging informational of patients, the pathological information including the distribution and characteristics of MVI can be accurately mapped to the corresponding images. This novel pathological examination technique is named as IDS. The major difference of IDS and WSI is the slide formats and sizes. IDS sufficiently takes advantage of macro-slide as its bridging vehicle of pathological and imaging information. 
The present study first reported the clinical utility of IDS in detecting MVI in HCC. In this study, we showed that IDS had a higher MVI detection rate than classical pathological examination approaches. In our study, the detection rate of MVI using IDS was significantly higher than conventional pathological approaches (63.7\% vs. $33.0 \%$ or $22.0 \%$ ), indicating that IDS had superior power in MVI detection compared with classical method.

Additionally, we provided evidence that in patients diagnosed with MVI negative status, the recurrence rate was significantly lower using IDS compared with conventional pathological method (6.1\% vs. $16.4 \%$ or $16.9 \%)$, suggesting it can guide postoperative surveillance and adjuvant treatments through screening those patients with MVI false-negative status (that is, MVI was detected by IDS but undetected by conventional pathological testing) in conventional pathological methods, thus reducing long-term recurrence rates. Furthermore, IDS and conventional pathological testing could complement with each other mutually. Our findings demonstrated that patients detected as MVI positive in both IDS and conventional pathological protocols were most likely to occur disease recurrence in the near future following initial hepatic surgery. The recurrence rate of patients with MVI false-negative status was substantially higher than that of patients with actual MVI negative status.

We also selected AFP as a suitable and robust biomarker to identify MVI false-negative patients in conventional pathological protocol. Approximately 70\% of HCC patients who were diagnosed as MVI negative status in conventional pathological examination may be MVI positive status in IDS testing. This encouraging result showed that IDS outperformed conventional pathological method in MVI detection for patients with abnormal AFP. For patients with normal AFP level ( $\leq 20 \mathrm{ng} / \mathrm{mL}$ ) and MVI negative status in conventional pathological method, IDS was also a crucial way to confirm the actual MVI status.

Previously, Sheng et al (13). proposed a standardized pathological proposal for evaluating MVI of HCC. They concluded that the MVI detection rate determined by seven-point sampling protocol (SPSP) was significantly higher than that determined by 3-point sampling method (47.1\% vs. 34.5\%, $P=0.048)$. Nevertheless, there was no marked difference in MVI detection rate between SPSP and 13-point sampling method $(47.1 \%$ vs. $51.3 \%, P=0.517)$. Therefore, we suppose that it is a futile effort to simply increase the sampling numbers beyond 7 points in conventional pathological slides. Contrarily, IDS is a useful and promising pathological technique to further increase $\mathrm{MVI}$ detection rates on the basis of conventional pathological testing method, thus guiding the subsequent treatment strategies of patients with MVI.

The potential application of IDS in clinical practice goes beyond MVI detection. In our on-going study, the locations of MVI can be accurately positioned using IDS, providing a reliable way to explore the distribution patterns of MVI in HCC and other tumors. Additionally, IDS can facilitate in judging the degree of tumor necrosis and observing the infiltration of inflammatory and immune cells, which can improve the efficacy evaluation of non-surgical treatments, such as transarterial chemoembolization, tyrosine kinase inhibitors and immune checkpoint inhibitors.

This study has some limitations. First, the small sample size and observational nature of this study may potentially affect the results. Second, all of the patients included in this study had a background of HBV 
infection. Whether IDS is applicable to patients with other etiologies of HCC needs further investigation. Third, this study is based upon our single-center data. The findings derived from this study require external validations.

\section{Conclusions}

This study demonstrates that imaging matching digital macro-slide (IDS) first implemented by our team can help improve the detection rate of MVI in $\mathrm{HCC}$ and refine the prediction of $\mathrm{HCC}$ prognosis. It highlights the importance of establishment of a novel pathological algorithm of IDS to study HCC more comprehensively and allow for a better understanding of number and distribution of MVI under microscope. In the future, combining with artificial intelligence-driven approaches, IDS has the opportunity to be the standardized pathological examination method in all types of cancers.

\section{Declarations}

\section{Conflict of interest:}

The authors have no conflicts of interest to declare.

\section{Data availability statement:}

Data sets analyzed in the study can be provided by the corresponding author on reasonable request.

\section{Ethics approval and consent to participate:}

This study was conducted in accordance with the Declaration of Helsinki (as revised in 2013) and approved by the Eastern Hepatobiliary Surgery Hospital (EHBH) Clinical Research Ethics Committee. All patients provided informed consent for data to be used for research purposes, and no personal information was disclosed.

\section{Role of the funding source:}

The Key Project of the National Natural Science Foundation of China (No: 81730097); the Grants of the Science Fund for Creative Research Groups (No: 81521091); the National Natural Science Foundation of China (No: 81602523, 82072618); and Shanghai Municipal Health Bureau (No: SHDC2020CR1004A).

\section{Authors contributions:}

Conception and design: Shu-Qun Cheng, Hui Dong, Hong-Ming Yu, Kang Wang, Jin-Kai Feng, Lei Lu; Administrative support: Shu-Qun Cheng;

Provision of study materials or patients: Yu-Qiang Cheng, Wei-Xing Guo, Jie Shi; 
Collection and assembly of data: Hong-Ming Yu, Kang Wang, Jin-Kai Feng, Lei Lu, Wen-Ming Cong;

Data analysis and interpretation: Hong-Ming Yu, Kang Wang, Jin-Kai Feng, Lei Lu, Wan Yee Lau;

Statistical analysis: Yu-Chen Qin;

Manuscript writing: All authors;

Final approval of manuscript: All authors.

\section{References}

1. Llovet JM, Kelley RK, Villanueva A, Singal AG, Pikarsky E, Roayaie S, et al. Hepatocellular carcinoma. Nat Rev Dis Primers. 2021;7(1):6.

2. Feng LH, Dong H, Lau WY, Yu H, Zhu YY, Zhao Y, et al. Novel microvascular invasion-based prognostic nomograms to predict survival outcomes in patients after R0 resection for hepatocellular carcinoma. $\mathrm{J}$ Cancer Res Clin Oncol. 2017;143(2):293-303.

3. Wang H, Wu MC, Cong WM. Microvascular invasion predicts a poor prognosis of solitary hepatocellular carcinoma up to $2 \mathrm{~cm}$ based on propensity score matching analysis. Hepatol Res. 2019;49(3):344-54.

4. Wang H, Qian YW, Wu MC, Cong WM. Liver Resection Is Justified in Patients with BCLC Intermediate Stage Hepatocellular Carcinoma without Microvascular Invasion. J Gastrointest Surg. 2020;24(12):273747.

5. Sumie S, Kuromatsu R, Okuda K, Ando E, Takata A, Fukushima N, et al. Microvascular invasion in patients with hepatocellular carcinoma and its predictable clinicopathological factors. Ann Surg Oncol. 2008;15(5):1375-82.

6. Mercan E, Mehta S, Bartlett J, Shapiro LG, Weaver DL, Elmore JG. Assessment of Machine Learning of Breast Pathology Structures for Automated Differentiation of Breast Cancer and High-Risk Proliferative Lesions. JAMA Netw Open. 2019;2(8):e198777.

7. Halama N, Zoernig I, Spille A, Michel S, Kloor M, Grauling-Halama S, et al. Quantification of prognostic immune cell markers in colorectal cancer using whole slide imaging tumor maps. Anal Quant Cytol Histol. 2010;32(6):333-40.

8. Wang H, Jiang Y, Li B, Cui Y, Li D, Li R. Single-Cell Spatial Analysis of Tumor and Immune Microenvironment on Whole-Slide Image Reveals Hepatocellular Carcinoma Subtypes. Cancers (Basel). 2020;12(12).

9. Dudek K, Kornasiewicz O, Remiszewski P, Kobryń K, Ziarkiewicz-Wróblewska B, Górnicka B, et al. Impact of tumor characteristic on the outcome of liver transplantation in patients with hepatocellular carcinoma. 
Transplant Proc. 2009;41(8):3135-7.

10. Huang ZY, Liang BY, Xiong M, Zhan DQ, Wei S, Wang GP, et al. Long-term outcomes of repeat hepatic resection in patients with recurrent hepatocellular carcinoma and analysis of recurrent types and their prognosis: a single-center experience in China. Ann Surg Oncol. 2012;19(8):2515-25.

11. Mazzaferro V, Llovet JM, Miceli R, Bhoori S, Schiavo M, Mariani L, et al. Predicting survival after liver transplantation in patients with hepatocellular carcinoma beyond the Milan criteria: a retrospective, exploratory analysis. Lancet Oncol. 2009;10(1):35-43.

12. Fan ST, Poon RT, Yeung C, Lam CM, Lo CM, Yuen WK, et al. Outcome after partial hepatectomy for hepatocellular cancer within the Milan criteria. Br J Surg. 2011;98(9):1292-300.

13. Sheng X, Ji Y, Ren GP, Lu CL, Yun JP, Chen LH, et al. A standardized pathological proposal for evaluating microvascular invasion of hepatocellular carcinoma: a multicenter study by LCPGC. Hepatol Int. 2020;14(6):1034-47.

\section{Tables}

Table 1. Baseline characteristics of patients with hepatocellular carcinoma. 


\begin{tabular}{|c|c|}
\hline Variables & Total $(n=91)$ \\
\hline \multicolumn{2}{|l|}{ Gender, n (\%) } \\
\hline Female & $13(14.29)$ \\
\hline Male & $78(85.71)$ \\
\hline Age(year), Mean \pm SD & $56.58 \pm 9.30$ \\
\hline \multicolumn{2}{|l|}{ HBsAg, n (\%) } \\
\hline Negative & $14(15.38)$ \\
\hline Positive & 77 (84.62) \\
\hline \multicolumn{2}{|l|}{ HBeAg, n (\%) } \\
\hline Negative & 74 (81.32) \\
\hline Positive & $17(18.68)$ \\
\hline \multicolumn{2}{|l|}{ HBcAb, n (\%) } \\
\hline Negative & $3(3.30)$ \\
\hline Positive & $88(96.70)$ \\
\hline \multicolumn{2}{|l|}{ HBV-DNA, n (\%) } \\
\hline$<1000$ copies/mL & $53(58.24)$ \\
\hline$\geq 1000$ copies $/ \mathrm{mL}$ & $38(41.76)$ \\
\hline \multicolumn{2}{|l|}{ HCV, n (\%) } \\
\hline Negative & $87(95.60)$ \\
\hline Positive & $4(4.40)$ \\
\hline AFP, Median (Q1, Q3), ug/L & $26.70(5.45,511.25)$ \\
\hline PIVKA-II, Median (Q1, Q3), mAU/mL & $274.00(62.50,1172.00)$ \\
\hline CA199, Median (Q1, Q3), U/mL & $15.50(8.65,28.60)$ \\
\hline CEA, Median (Q1, Q3), ug/L & $2.60(1.70,3.40)$ \\
\hline ALT, Median (Q1, Q3), U/L & $25.00(16.00,44.00)$ \\
\hline AST, Median (Q1, Q3), U/L & $25.00(18.00,38.00)$ \\
\hline Total bilirubin, Median (Q1, Q3), umol/L & $14.30(10.85,18.10)$ \\
\hline GGT, Median (Q1, Q3), U/L & $39.00(25.00,77.00)$ \\
\hline Albumin, Mean \pm SD, g/L & $42.90 \pm 4.45$ \\
\hline
\end{tabular}




\begin{tabular}{|c|c|}
\hline GLU, Median (Q1, Q3), mmol/L & $5.12(4.79,5.65)$ \\
\hline ALP, Median (Q1, Q3), U/L & $77.00(59.50,98.00)$ \\
\hline WBC, Median (Q1, Q3), 10/L & $4.81(3.73,5.72)$ \\
\hline $\mathrm{RBC}$, Mean $\pm \mathrm{SD}, 10^{12} / \mathrm{L}$ & $4.57 \pm 0.47$ \\
\hline HGB, Mean $\pm S D, g / L$ & $140.80 \pm 15.47$ \\
\hline PLT, Mean $\pm \mathrm{SD}, 10^{9} / \mathrm{L}$ & $149.08 \pm 61.11$ \\
\hline PT, Median (Q1, Q3), s & $11.50(11.00,12.05)$ \\
\hline Tumor size, Median (Q1, Q3), cm & $3.80(2.50,5.50)$ \\
\hline \multicolumn{2}{|l|}{ Tumor number, n (\%) } \\
\hline 1 & $83(91.21)$ \\
\hline 2 & $7(7.69)$ \\
\hline 4 & $1(1.10)$ \\
\hline \multicolumn{2}{|l|}{ PVTT, n (\%) } \\
\hline Absence & $87(95.60)$ \\
\hline Presence & $4(4.40)$ \\
\hline \multicolumn{2}{|l|}{ Encapsulation, n (\%) } \\
\hline No & $23(25.27)$ \\
\hline Incomplete & $42(46.15)$ \\
\hline Complete & $26(28.57)$ \\
\hline \multicolumn{2}{|l|}{ Liver cirrhosis, n (\%) } \\
\hline No & $38(41.76)$ \\
\hline Yes & $53(58.24)$ \\
\hline Child-Pugh class A, n (\%) & $91(100.00)$ \\
\hline \multicolumn{2}{|l|}{ Recurrence, n (\%) } \\
\hline No & $67(73.62)$ \\
\hline Yes & 24 (26.37) \\
\hline
\end{tabular}

Abbreviations: HBsAg: hepatitis B surface antigen; HBeAg: hepatitis B e antigen; HBcAb: hepatitis B core antibody; HBV-DNA: hepatitis B virus-deoxyribonucleic acid; HCV: hepatitis C virus; AFP: alpha-fetoprotein; PIVKA-II: protein induced by vitamin K antagonist-II; CA199: carbohydrate antigen199; CEA: 
carcinoembryonic antigen; ALT: alanine aminotransferase; AST: aspartate aminotransferase; GGT: $\gamma^{-}$ glutamyltransferase; GLU: glucose; ALP: alkaline phosphatase; WBC: white blood cells; RBC: red blood cells; PLT: platelet; PT: prothrombin time; PVTT: portal vein tumor thrombus.

Table 2. MVI sensitivity and specificity under 3-Piont, 7-Point and IDS.

\begin{tabular}{|lll|}
\hline Metrics & 3-Point & 7-Point \\
\hline Accuracy & $0.58(0.47-0.68)$ & $0.69(0.59-0.78)$ \\
\hline Sensitivity & $0.34(0.22-0.48)$ & $0.52(0.38-0.65)$ \\
\hline Specificity & $1.00(0.89-1.00)$ & $1.00(0.89-1.00)$ \\
\hline Positive Predictive Value & $1.00(0.83-1.00)$ & $1.00(0.88-1.00)$ \\
\hline Negative Predictive Value & $0.46(0.35-0.59)$ & $0.54(0.41-0.67)$ \\
\hline
\end{tabular}

Abbreviations: MVl: microvascular invasion; 3-Point: 3-point baseline sampling protocol; 7-Point: 7-point baseline sampling protocol; IDS: image-matching digital macro-slide.

Table 3. The clinicopathological features were compared in different combinations of 3-Point and IDS with MVI or not. 


\begin{tabular}{|c|c|c|c|c|}
\hline Variables & $\begin{array}{l}\text { 3- } \\
\text { Point(-)IDS(-) (n } \\
=33)\end{array}$ & $\begin{array}{l}\text { 3- } \\
\text { Point(-)IDS }(+)(n \\
=38)\end{array}$ & $\begin{array}{l}\text { 3- } \\
\text { Point }(+) \operatorname{IDS}(+)(\mathrm{n} \\
=20)\end{array}$ & P \\
\hline Gender, n (\%) & & & & 0.690 \\
\hline Female & $4(30.77)$ & $7(53.85)$ & $2(15.38)$ & \\
\hline Male & $29(37.18)$ & $31(39.74)$ & $18(23.08)$ & \\
\hline Age(year), Mean \pm SD & $57.09 \pm 8.96$ & $56.84 \pm 9.50$ & $55.25 \pm 9.80$ & 0.768 \\
\hline HBsAg, n (\%) & & & & 0.603 \\
\hline Negative & $7(50.00)$ & $5(35.71)$ & $2(14.29)$ & \\
\hline Positive & $26(33.77)$ & $33(42.86)$ & $18(23.38)$ & \\
\hline HBeAg, n (\%) & & & & 0.654 \\
\hline Negative & $28(37.84)$ & $29(39.19)$ & $17(22.97)$ & \\
\hline Positive & $5(29.41)$ & $9(52.94)$ & $3(17.65)$ & \\
\hline HBcAb, n (\%) & & & & 0.603 \\
\hline Negative & $2(66.67)$ & $1(33.33)$ & $0(0.00)$ & \\
\hline Positive & $31(35.23)$ & $37(42.05)$ & $20(22.73)$ & \\
\hline HBV-DNA, n (\%) & & & & 0.921 \\
\hline \1000 copies/mL & $20(37.74)$ & $22(41.51)$ & $11(20.75)$ & \\
\hline$\geq 1000$ copies $/ \mathrm{mL}$ & $13(34.21)$ & $16(42.11)$ & $9(23.68)$ & \\
\hline HCV, n (\%) & & & & 0.826 \\
\hline Negative & $31(35.63)$ & $37(42.53)$ & $19(21.84)$ & \\
\hline Positive & $2(50.00)$ & $1(25.00)$ & $1(25.00)$ & \\
\hline AFP, Median (Q1, Q3), ug/L & $\begin{array}{l}6.10(3.10 \\
20.30)\end{array}$ & $\begin{array}{l}81.30(10.12 \\
560.65)\end{array}$ & $\begin{array}{l}346.35(31.43, \\
1210.00)\end{array}$ & $\begin{array}{l}<.001 \\
0.01\end{array}$ \\
\hline $\begin{array}{l}\text { PIVKA- II, Median (Q1, Q3), } \\
\text { mAU/mL }\end{array}$ & $\begin{array}{l}107.00(33.00 \\
412.00)\end{array}$ & $\begin{array}{l}449.00(90.50 \\
3168.75)\end{array}$ & $\begin{array}{l}290.50(137.50 \\
866.25)\end{array}$ & 0.032 \\
\hline $\begin{array}{l}\text { CA199, Median (Q1, Q3), } \\
\text { U/mL }\end{array}$ & $\begin{array}{l}14.00(6.60 \\
21.10)\end{array}$ & $\begin{array}{l}17.50(9.50 \\
28.52)\end{array}$ & $\begin{array}{l}18.00(9.35 \\
36.15)\end{array}$ & 0.263 \\
\hline CEA, Median (Q1, Q3), ug/L & $2.80(2.00,3.70)$ & $2.70(1.90,3.27)$ & $1.70(1.37,3.23)$ & 0.168 \\
\hline ALT, Median (Q1, Q3), U/L & $\begin{array}{l}25.00(15.00 \\
50.00)\end{array}$ & $\begin{array}{l}20.50(14.25 \\
33.75)\end{array}$ & $\begin{array}{l}27.50(21.50 \\
54.25)\end{array}$ & 0.199 \\
\hline AST, Median (Q1, Q3), U/L & $\begin{array}{l}25.00(17.00 \\
36.00)\end{array}$ & $\begin{array}{l}21.50(18.00 \\
37.50)\end{array}$ & $\begin{array}{l}32.50(23.00 \\
39.00)\end{array}$ & 0.222 \\
\hline
\end{tabular}




\begin{tabular}{|c|c|c|c|c|}
\hline $\begin{array}{l}\text { Total bilirubin, Median (Q1, } \\
\text { Q3), umol/L }\end{array}$ & $\begin{array}{l}15.30(12.20 \\
18.10)\end{array}$ & $\begin{array}{l}\text { 14.10 (11.57, } \\
17.53)\end{array}$ & $\begin{array}{l}14.05(9.50 \\
20.38)\end{array}$ & 0.788 \\
\hline GGT, Median (Q1, Q3), U/L & $\begin{array}{l}38.00(25.00 \\
71.00)\end{array}$ & $\begin{array}{l}32.00(24.00 \\
61.75)\end{array}$ & $\begin{array}{l}52.00(39.25 \\
103.00)\end{array}$ & 0.069 \\
\hline Albumin, Mean \pm SD, g/L & $43.09 \pm 3.74$ & $42.32 \pm 4.65$ & $43.71 \pm 5.15$ & 0.505 \\
\hline $\begin{array}{l}\text { GLU, Median (Q1, Q3), } \\
\mathrm{mmol} / \mathrm{L}\end{array}$ & $5.24(4.89,6.07)$ & $5.03(4.67,5.44)$ & $5.29(5.01,5.95)$ & 0.231 \\
\hline ALP, Median (Q1, Q3), U/L & $\begin{array}{l}79.00(63.00 \\
92.00)\end{array}$ & $\begin{array}{l}64.50(54.25 \\
79.25)\end{array}$ & $\begin{array}{l}89.50(72.25 \\
103.50)\end{array}$ & 0.017 \\
\hline WBC, Median (Q1, Q3), 109/L & $4.87(3.97,6.26)$ & $4.81(3.73,5.64)$ & $4.54(3.29,5.74)$ & 0.587 \\
\hline $\mathrm{RBC}$, Mean $\pm \mathrm{SD}, 10^{12} / \mathrm{L}$ & $4.53 \pm 0.48$ & $4.60 \pm 0.42$ & $4.60 \pm 0.57$ & 0.779 \\
\hline HGB, Mean $\pm S D, g / L$ & $141.33 \pm 14.19$ & $140.58 \pm 16.48$ & $140.35 \pm 16.25$ & 0.969 \\
\hline PLT, Mean \pm SD, $10^{9} / \mathrm{L}$ & $159.39 \pm 71.71$ & $149.55 \pm 54.17$ & $131.15 \pm 52.85$ & 0.267 \\
\hline PT, Median (Q1, Q3), s & $\begin{array}{l}11.50(10.90 \\
11.90)\end{array}$ & $\begin{array}{l}11.50(11.00 \\
12.12)\end{array}$ & $\begin{array}{l}11.60(11.07 \\
12.15)\end{array}$ & 0.697 \\
\hline $\begin{array}{l}\text { Tumor size, Median (Q1, Q3), } \\
\text { cm }\end{array}$ & $3.50(2.50,4.30)$ & $3.65(2.08,6.12)$ & $5.00(3.50,6.08)$ & 0.127 \\
\hline Tumor number, $\mathrm{n}(\%)$ & & & & 0.009 \\
\hline 1 & $33(39.76)$ & $35(42.17)$ & $15(18.07)$ & \\
\hline 2 & $0(0.00)$ & $3(42.86)$ & $4(57.14)$ & \\
\hline 4 & $0(0.00)$ & $0(0.00)$ & $1(100.00)$ & \\
\hline PVTT, n (\%) & & & & 0.203 \\
\hline Absence & $33(37.93)$ & $36(41.38)$ & $18(20.69)$ & \\
\hline Presence & $0(0.00)$ & $2(50.00)$ & $2(50.00)$ & \\
\hline Encapsulation, n (\%) & & & & 0.195 \\
\hline No & $4(17.39)$ & $13(56.52)$ & $6(26.09)$ & \\
\hline Incomplete & $16(38.10)$ & $16(38.10)$ & $10(23.81)$ & \\
\hline Complete & $13(50.00)$ & $9(34.62)$ & $4(15.38)$ & \\
\hline Liver cirrhosis, n (\%) & & & & 0.624 \\
\hline No & $13(34.21)$ & 18 (47.37) & $7(18.42)$ & \\
\hline Yes & $20(37.74)$ & $20(37.74)$ & $13(24.53)$ & \\
\hline Child-Pugh class A, n (\%) & $33(36.26)$ & $38(41.76)$ & $20(21.98)$ & 1.000 \\
\hline
\end{tabular}


Abbreviations: 3-Point: 3-point baseline sampling protocol; MVI: microvascular invasion; IDS: imagematching digital macro-slide; HBsAg: hepatitis B surface antigen; HBeAg: hepatitis B e antigen; HBcAb: hepatitis B core antibody; HBV-DNA: hepatitis B virus-deoxyribonucleic acid; HCV: hepatitis C virus; AFP: alpha-fetoprotein; PIVKA-II: protein induced by vitamin K antagonist-II; CA199: carbohydrate antigen199; CEA: carcinoembryonic antigen; ALT: alanine aminotransferase; AST: aspartate aminotransferase; GGT: Y-glutamyltransferase; GLU: glucose; ALP: alkaline phosphatase; WBC: white blood cells; RBC: red blood cells; PLT: platelet; PT: prothrombin time; PVTT: portal vein tumor thrombus.

Table4. The clinicopathological features were compared in different combinations of 7-Point and IDS with MVI or not. 


\begin{tabular}{|c|c|c|c|c|}
\hline Variables & $\begin{array}{l}7 \mathrm{P}(-) \mathrm{MS}(-)(\mathrm{n}= \\
33)\end{array}$ & $\begin{array}{l}7 P(-) M S(+)(n= \\
28)\end{array}$ & $\begin{array}{l}7 \mathrm{P}(+) M S(+)(n= \\
30)\end{array}$ & $P$ \\
\hline Gender, n (\%) & & & & 0.147 \\
\hline Female & $4(30.77)$ & $7(53.85)$ & $2(15.38)$ & \\
\hline Male & $29(37.18)$ & $21(26.92)$ & $28(35.90)$ & \\
\hline Age(year), Mean \pm SD & $57.09 \pm 8.96$ & $57.32 \pm 10.30$ & $55.33 \pm 8.86$ & 0.670 \\
\hline HBsAg, n (\%) & & & & 0.474 \\
\hline Negative & $7(50.00)$ & $4(28.57)$ & $3(21.43)$ & \\
\hline Positive & 26 (33.77) & 24 (31.17) & 27 (35.06) & \\
\hline HBeAg, n (\%) & & & & 0.267 \\
\hline Negative & $28(37.84)$ & $20(27.03)$ & $26(35.14)$ & \\
\hline Positive & $5(29.41)$ & $8(47.06)$ & $4(23.53)$ & \\
\hline HBcAb, n (\%) & & & & 0.641 \\
\hline Negative & $2(66.67)$ & $1(33.33)$ & $0(0.00)$ & \\
\hline Positive & $31(35.23)$ & 27 (30.68) & 30 (34.09) & \\
\hline HBV-DNA, n (\%) & & & & 0.942 \\
\hline \1000 copies/mL & 20 (37.74) & $16(30.19)$ & 17 (32.08) & \\
\hline$\geq 1000$ copies $/ \mathrm{mL}$ & $13(34.21)$ & $12(31.58)$ & $13(34.21)$ & \\
\hline HCV, n (\%) & & & & 0.458 \\
\hline Negative & $31(35.63)$ & $26(29.89)$ & 30 (34.48) & \\
\hline Positive & $2(50.00)$ & $2(50.00)$ & $0(0.00)$ & \\
\hline AFP, Median (Q1, Q3), ug/L & $\begin{array}{l}6.10(3.10 \\
20.30)\end{array}$ & $\begin{array}{l}160.55(20.92 \\
1210.00)\end{array}$ & $\begin{array}{l}66.35(13.67 \\
1210.00)\end{array}$ & $\begin{array}{l}< \\
0.001\end{array}$ \\
\hline $\begin{array}{l}\text { PIVKA-II, Median (Q1, Q3), } \\
\mathrm{mAU} / \mathrm{mL}\end{array}$ & $\begin{array}{l}107.00(33.00 \\
412.00)\end{array}$ & $\begin{array}{l}460.50(239.25 \\
3084.25)\end{array}$ & $\begin{array}{l}257.00(90.50 \\
725.75)\end{array}$ & 0.021 \\
\hline $\begin{array}{l}\text { CA199, Median (Q1, Q3), } \\
\text { U/mL }\end{array}$ & $\begin{array}{l}14.00(6.60 \\
21.10)\end{array}$ & $\begin{array}{l}19.50(11.17 \\
30.35)\end{array}$ & $\begin{array}{l}15.40(8.62 \\
33.60)\end{array}$ & 0.336 \\
\hline CEA, Median (Q1, Q3), ug/L & $2.80(2.00,3.70)$ & $2.70(2.08,3.12)$ & $1.90(1.33,3.53)$ & 0.170 \\
\hline ALT, Median (Q1, Q3), U/L & $\begin{array}{l}25.00(15.00 \\
50.00)\end{array}$ & $\begin{array}{l}22.50(16.25 \\
36.50)\end{array}$ & $\begin{array}{l}25.00(17.25 \\
46.75)\end{array}$ & 0.853 \\
\hline AST, Median (Q1, Q3), U/L & $\begin{array}{l}25.00(17.00 \\
36.00)\end{array}$ & $\begin{array}{l}24.00(18.00 \\
39.50)\end{array}$ & $\begin{array}{l}26.50(18.25 \\
37.75)\end{array}$ & 0.936 \\
\hline
\end{tabular}




\begin{tabular}{|c|c|c|c|c|}
\hline $\begin{array}{l}\text { Total bilirubin, Median (Q1, } \\
\text { Q3), umol/L }\end{array}$ & $\begin{array}{l}15.30(12.20 \\
18.10)\end{array}$ & $\begin{array}{l}13.55(11.38 \\
17.88)\end{array}$ & $\begin{array}{l}15.05(9.85 \\
19.00)\end{array}$ & 0.789 \\
\hline GGT, Median (Q1, Q3), U/L & $\begin{array}{l}38.00(25.00 \\
71.00)\end{array}$ & $\begin{array}{l}32.00(24.00 \\
63.00)\end{array}$ & $\begin{array}{l}45.00(30.50 \\
86.00)\end{array}$ & 0.471 \\
\hline Albumin, Mean $\pm S D, g / L$ & $43.09 \pm 3.74$ & $41.79 \pm 4.70$ & $43.74 \pm 4.84$ & 0.242 \\
\hline $\begin{array}{l}\text { GLU, Median (Q1, Q3), } \\
\mathrm{mmol} / \mathrm{L}\end{array}$ & $5.24(4.89,6.07)$ & $5.21(4.94,5.68)$ & $5.02(4.75,5.40)$ & 0.392 \\
\hline ALP, Median (Q1, Q3), U/L & $\begin{array}{l}79.00(63.00 \\
92.00)\end{array}$ & $\begin{array}{l}66.50(54.75 \\
83.00)\end{array}$ & $\begin{array}{l}79.00(60.75 \\
101.75)\end{array}$ & 0.246 \\
\hline WBC, Median (Q1, Q3), 10\%/L & $4.87(3.97,6.26)$ & $4.43(3.43,5.54)$ & $5.02(3.66,5.64)$ & 0.473 \\
\hline $\mathrm{RBC}$, Mean $\pm \mathrm{SD}, 10^{12} / \mathrm{L}$ & $4.53 \pm 0.48$ & $4.56 \pm 0.41$ & $4.64 \pm 0.52$ & 0.611 \\
\hline $\mathrm{HGB}$, Mean $\pm \mathrm{SD}, \mathrm{g} / \mathrm{L}$ & $141.33 \pm 14.19$ & $139.46 \pm 15.06$ & $141.47 \pm 17.50$ & 0.862 \\
\hline PLT, Mean $\pm \mathrm{SD}, 10^{9} / \mathrm{L}$ & $159.39 \pm 71.71$ & $144.00 \pm 57.54$ & $142.47 \pm 51.41$ & 0.481 \\
\hline PT, Median (Q1, Q3), s & $\begin{array}{l}11.50(10.90 \\
11.90)\end{array}$ & $\begin{array}{l}11.55(11.15 \\
12.70)\end{array}$ & $\begin{array}{l}11.45(11.00 \\
11.88)\end{array}$ & 0.610 \\
\hline $\begin{array}{l}\text { Tumor size, Median (Q1, Q3), } \\
\mathrm{cm}\end{array}$ & $3.50(2.50,4.30)$ & $4.00(2.45,6.32)$ & $4.65(2.73,6.00)$ & 0.254 \\
\hline Tumor number, n (\%) & & & & 0.053 \\
\hline 1 & $33(39.76)$ & $24(28.92)$ & $26(31.33)$ & \\
\hline 2 & $0(0.00)$ & $4(57.14)$ & $3(42.86)$ & \\
\hline 4 & $0(0.00)$ & $0(0.00)$ & $1(100.00)$ & \\
\hline PVTT, n (\%) & & & & 0.153 \\
\hline Absence & $33(37.93)$ & $27(31.03)$ & $27(31.03)$ & \\
\hline Presence & $0(0.00)$ & $1(25.00)$ & $3(75.00)$ & \\
\hline Encapsulation, n (\%) & & & & 0.181 \\
\hline No & $4(17.39)$ & $8(34.78)$ & $11(47.83)$ & \\
\hline Incomplete & $16(38.10)$ & $13(30.95)$ & $13(30.95)$ & \\
\hline Complete & $13(50.00)$ & $7(26.92)$ & $6(23.08)$ & \\
\hline Liver cirrhosis, n (\%) & & & & 0.942 \\
\hline No & $13(34.21)$ & $12(31.58)$ & $13(34.21)$ & \\
\hline Yes & $20(37.74)$ & $16(30.19)$ & $17(32.08)$ & \\
\hline Child-Pugh class A, n (\%) & $33(36.26)$ & $28(30.77)$ & 30 (32.97) & 1.000 \\
\hline
\end{tabular}


Abbreviations: 7-Point: 7-point baseline sampling protocol; MVI: microvascular invasion; IDS: image matching digital macro-slide; HBsAg: hepatitis $B$ surface antigen; HBeAg: hepatitis $B$ e antigen; $\mathrm{HBcAb:}$ hepatitis B core antibody; HBV-DNA: hepatitis B virus-deoxyribonucleic acid; HCV: hepatitis C virus; AFP: alpha-fetoprotein; PIVKA-II: protein induced by vitamin K antagonist-II; CA199: carbohydrate antigen199; CEA: carcinoembryonic antigen; ALT: alanine aminotransferase; AST: aspartate aminotransferase; GGT: Y-glutamyltransferase; GLU: glucose; ALP: alkaline phosphatase; WBC: white blood cells; RBC: red blood cells; PLT: platelet; PT: prothrombin time; PVTT: portal vein tumor thrombus.

Table 5. The cutoff value of AFP and PIVKA-II to detect MVI in 3-Point and 7-Piont baseline sampling protocol

\begin{tabular}{|lllll|}
\hline & 3-Point & & 7-Point & \\
\hline Metrics & $\begin{array}{l}\text { AFP Optimal } \\
\text { Cutoff } \geq 22.5\end{array}$ & $\begin{array}{l}\text { PIVKA-II Optimal } \\
\text { Cutoff } \geq 267\end{array}$ & $\begin{array}{l}\text { AFP Optimal } \\
\text { Cutoff } \geq 23.9\end{array}$ & $\begin{array}{l}\text { PIVKA-II Optimal } \\
\text { Cutoff } \geq 267\end{array}$ \\
\hline Accuracy & $0.73(0.61-0.83)$ & $0.66(0.54-0.77)$ & $0.77(0.65-0.87)$ & $0.70(0.57-0.81)$ \\
\hline Sensitivity & $0.68(0.51-0.82)$ & $0.66(0.49-0.80)$ & $0.75(0.55-0.89)$ & $0.75(0.55-0.89)$ \\
\hline Specificity & $0.79(0.61-0.91)$ & $0.67(0.48-0.82)$ & $0.79(0.61-0.91)$ & $0.67(0.48-0.82)$ \\
\hline $\begin{array}{l}\text { Positive } \\
\text { Predictive Value }\end{array}$ & $0.79(0.61-0.91)$ & $0.69(0.52-0.84)$ & $0.75(0.55-0.89)$ & $0.66(0.47-0.81)$ \\
\hline $\begin{array}{l}\text { Negative } \\
\text { Predictive Value }\end{array}$ & $0.68(0.51-0.82)$ & $0.63(0.45-0.79)$ & $0.79(0.61-0.91)$ & $0.76(0.56-0.90)$ \\
\hline
\end{tabular}

Abbreviations: AFP: alpha-fetoprotein; PIVKA-II: protein induced by vitamin K antagonist-Il; MVI: microvascular invasion; 3-Point: 3-point baseline sampling protocol; 7-Point: 7-point baseline sampling protocol.

Table 6. AFP normal value and cutoff value to distinguish MVI false negative status under 3-point baseline sampling protocol.

\begin{tabular}{|llllll|}
\hline Variables & Total $(\mathrm{n}=71)$ & MVI-3P(-)IDS $(-)(\mathrm{n}=33)$ & MVI-3P(-)IDS $(+)(\mathrm{n}=38)$ & $\mathrm{P}$ & Statistic \\
$\begin{array}{l}\text { AFP, } n \\
(\%)\end{array}$ & & & & 0.001 & 10.375 \\
$<20$ & $36(50.70)$ & $24(66.67)$ & $12(33.33)$ & & \\
$\geq 20$ & $35(49.30)$ & $9(25.71)$ & $26(74.29)$ & 0.000 & 12.433 \\
$\begin{array}{l}\text { AFP, } n \\
(\%)\end{array}$ & & & & & \\
$<22.5$ & $39(54.93)$ & $26(66.67)$ & $13(33.33)$ & & \\
$\geq 22.5$ & $32(45.07)$ & $7(21.88)$ & $25(78.12)$ & & \\
\hline
\end{tabular}


Abbreviations: AFP: alpha-fetoprotein; MVl: microvascular invasion; 3P: 3-point baseline sampling protocol; IDS: Image-matching digital macro-slide.

Table 7. AFP normal value and cutoff value to distinguish MVI false negative status under 7-point baseline sampling protocol.

\begin{tabular}{|llllll|}
\hline Variables & Total $(\mathrm{n}=61)$ & MVI-7P(-)IDS(-)(n=33) & MVI-7P(-)IDS(+) $(\mathrm{n}=28)$ & $\mathrm{P}$ & Statistic \\
\hline AFP, $\mathrm{n}(\%)$ & & & 0.000 & 11.962 \\
\hline$<20$ & $31(50.82)$ & $24(77.42)$ & $7(22.58)$ & & \\
\hline$\geq 20$ & $30(49.18)$ & $9(30.00)$ & $21(70.00)$ & & \\
\hline AFP, $n(\%)$ & & & & & \\
\hline$<23.9$ & $33(54.10)$ & $26(78.79)$ & $7(21.21)$ & & \\
\hline$\geq 23.9$ & $28(45.90)$ & $7(25.00)$ & $21(75.00)$ & \\
\hline
\end{tabular}

Abbreviations: AFP: alpha-fetoprotein; MVl: microvascular invasion; 7P: 7-point baseline sampling protocol; IDS: Image-matching digital macro-slide.

\section{Figures}

A
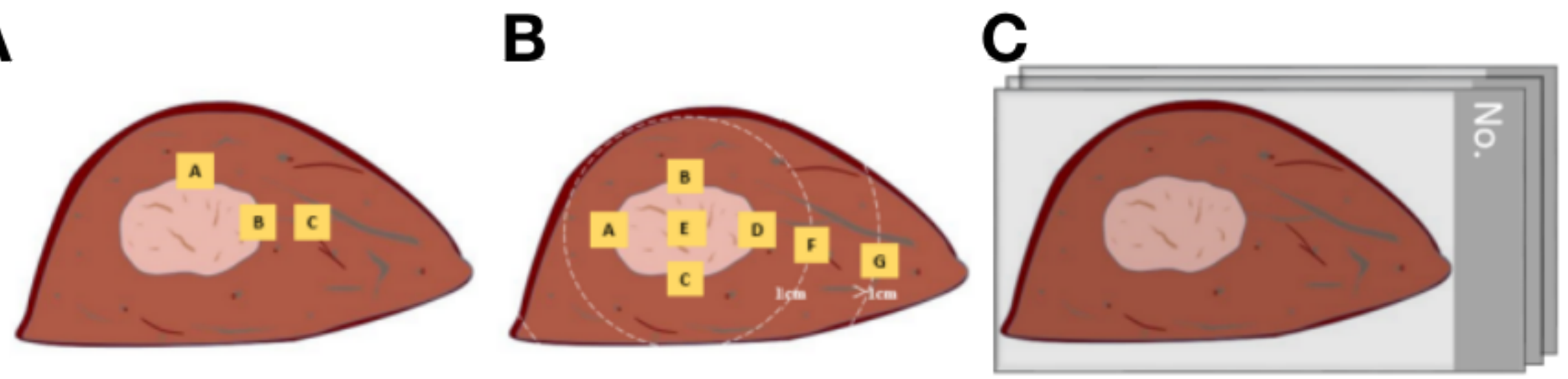

Figure 1

The model of different pathological sampling methods: A, 3-Point baseline sampling protocol. B, 7-Point baseline sampling protocol. C, IDS sampling protocol. 


\section{A}
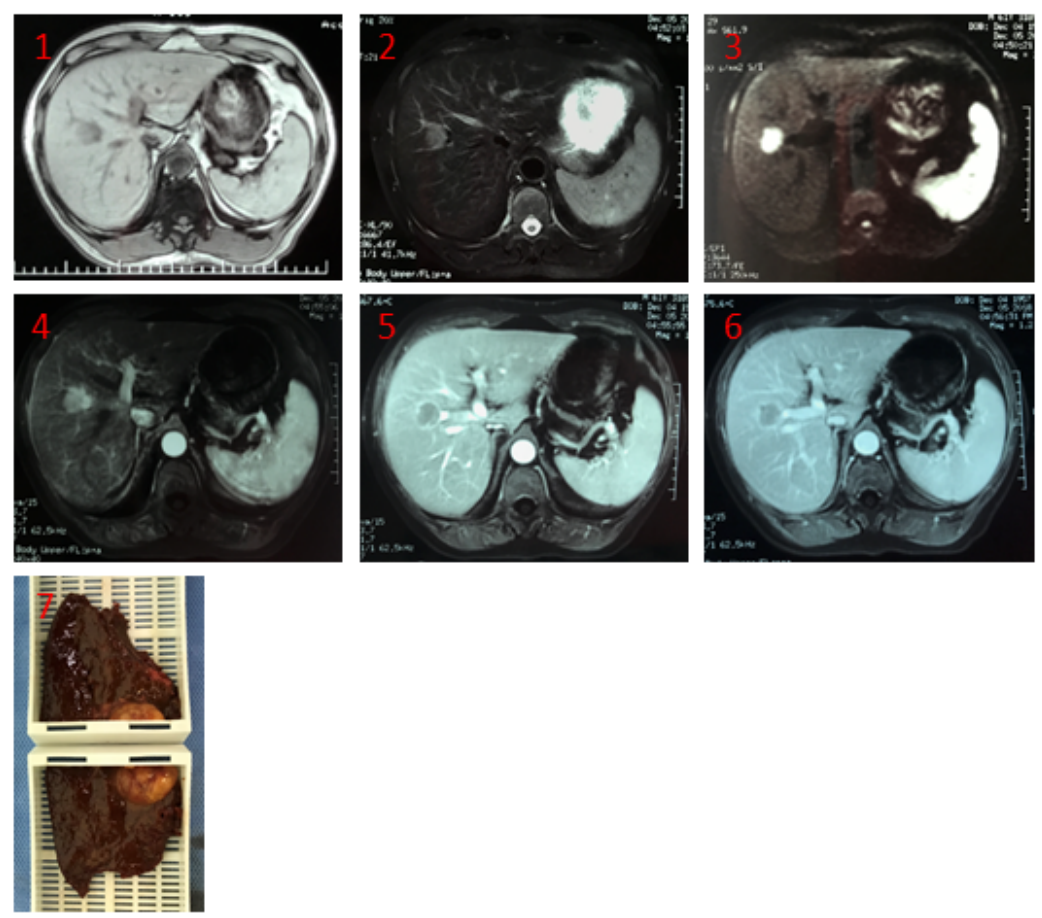

B
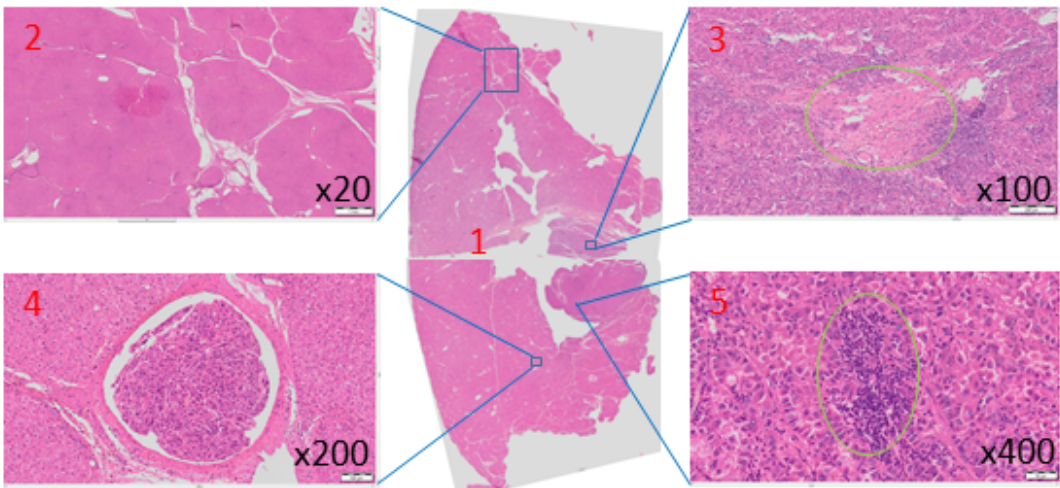

1

C

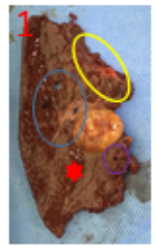

The former branch of RAPV

The former branch of Posterior segmental artery

The after branch of Posterior segmental artery

* The after branch of RAPV- MVI
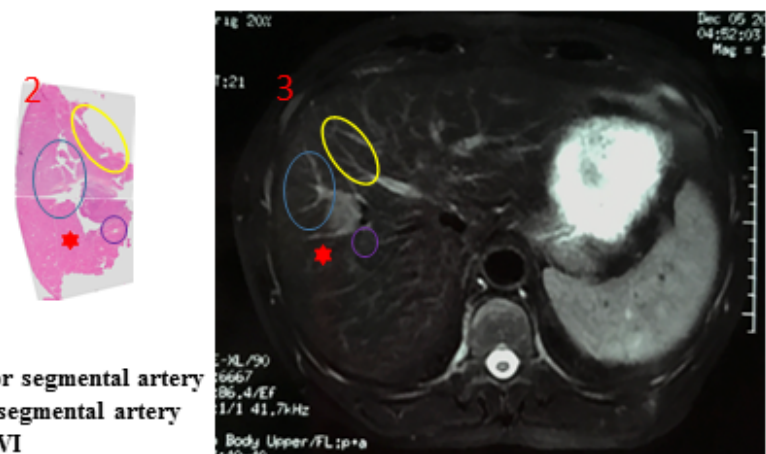

\section{Figure 2}

A case of image-matching digital macro-slide. A, Preoperative MRI and sample selection of the patient: 1, T1Wl; 2, T2Wl; 3, DWl; 4, arterial phase; 5, portal phase; 6 , delay phase. B, image after WSI scanning: 1 , making macro-slide samples; 2 , the overall view of the image after the sample is scanned and stitched; 3 , mixed nodular cirrhosis was observed after magnification of 20 times; 4, local hemorrhagic necrosis was seen inside the tumor after magnification of 100 times; 5, MVI was observed after magnification of 200 
times; 6 , accumulation of inflammatory cells in the tumor was observed after 400 -fold magnification. C, corresponding specimen and digital macro-slide combined to MRI imaging (T2WI): 1, specimen; 2, digital macro-slide; $3, \mathrm{MRI}$ imaging (T2WI), in 1: 1: 1. Marking the different vessels and MVI locations with different colors in the panel.

A

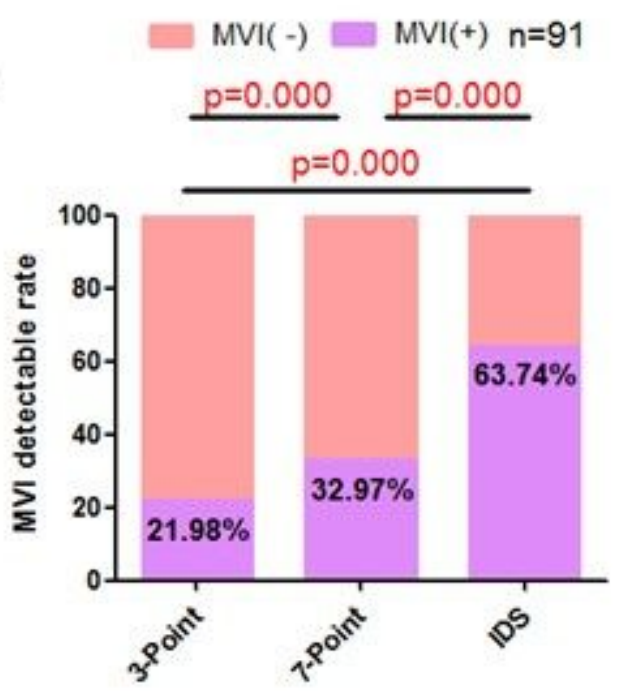

B

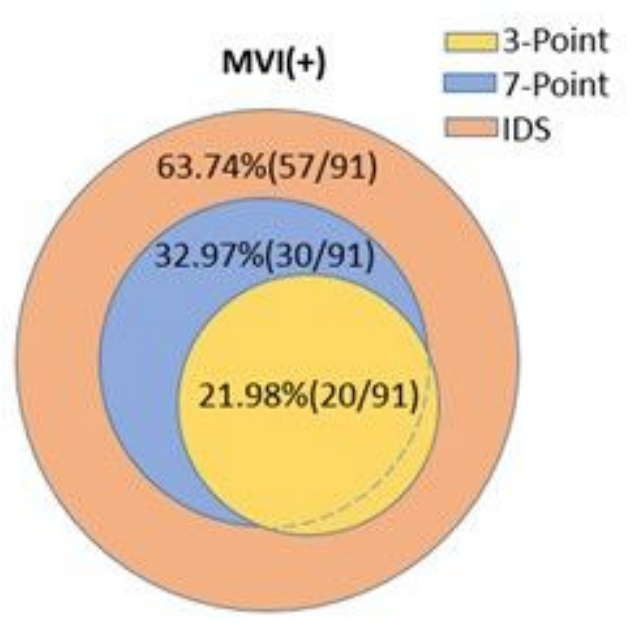

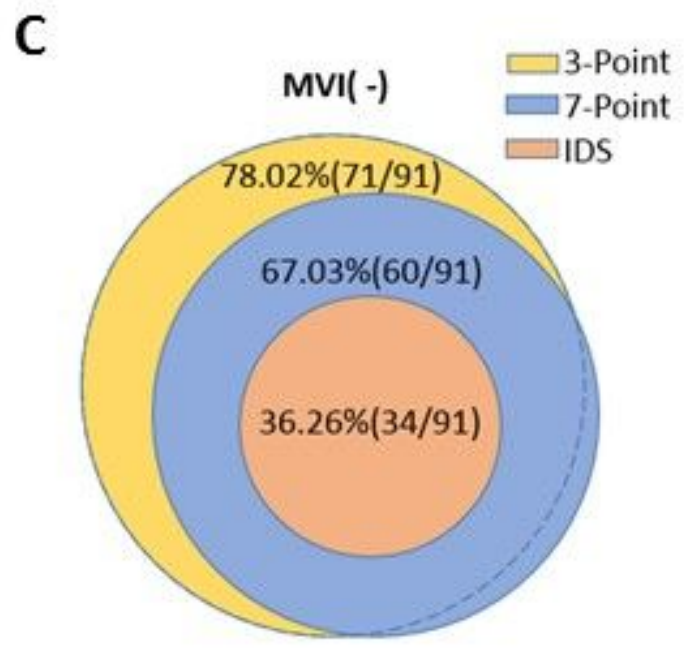

\section{Figure 3}

A, MVI detection rate under 3-point baseline sampling protocol, 7-point baseline sampling protocol and image matching digital macro-slide. B\&C, the composition of $\mathrm{MVI}$ positive and negative status patients in different detective methods (3-point baseline sampling protocol, 7-point baseline sampling protocol and image matching digital macro-slide). 

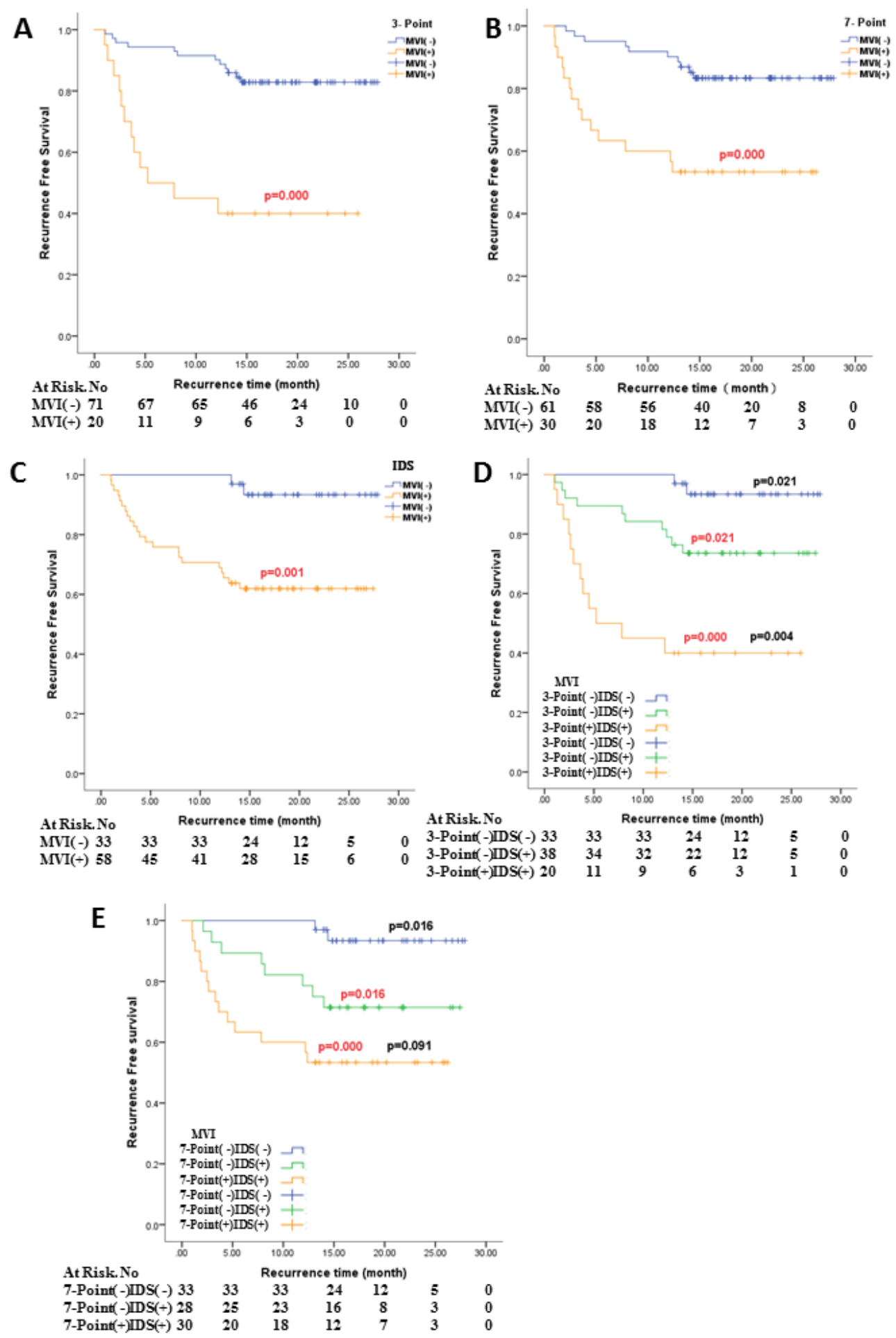

\section{Figure 4}

MVI related recurrence-free survival. A, MVI detected by 3-point baseline sampling protocol and related recurrence-free survival. B, MVI detected by 7-point baseline sampling protocol and related recurrence-free survival. C, MVI detected by image matching digital macro-slide and related recurrence-free survival. D, 3point baseline sampling protocol combined with image matching digital macro-slide to detect MVI and 
related recurrence-free survival. E, 7-point baseline sampling protocol combined with image matching digital macro-slide to detect MVI and related recurrence-free survival.
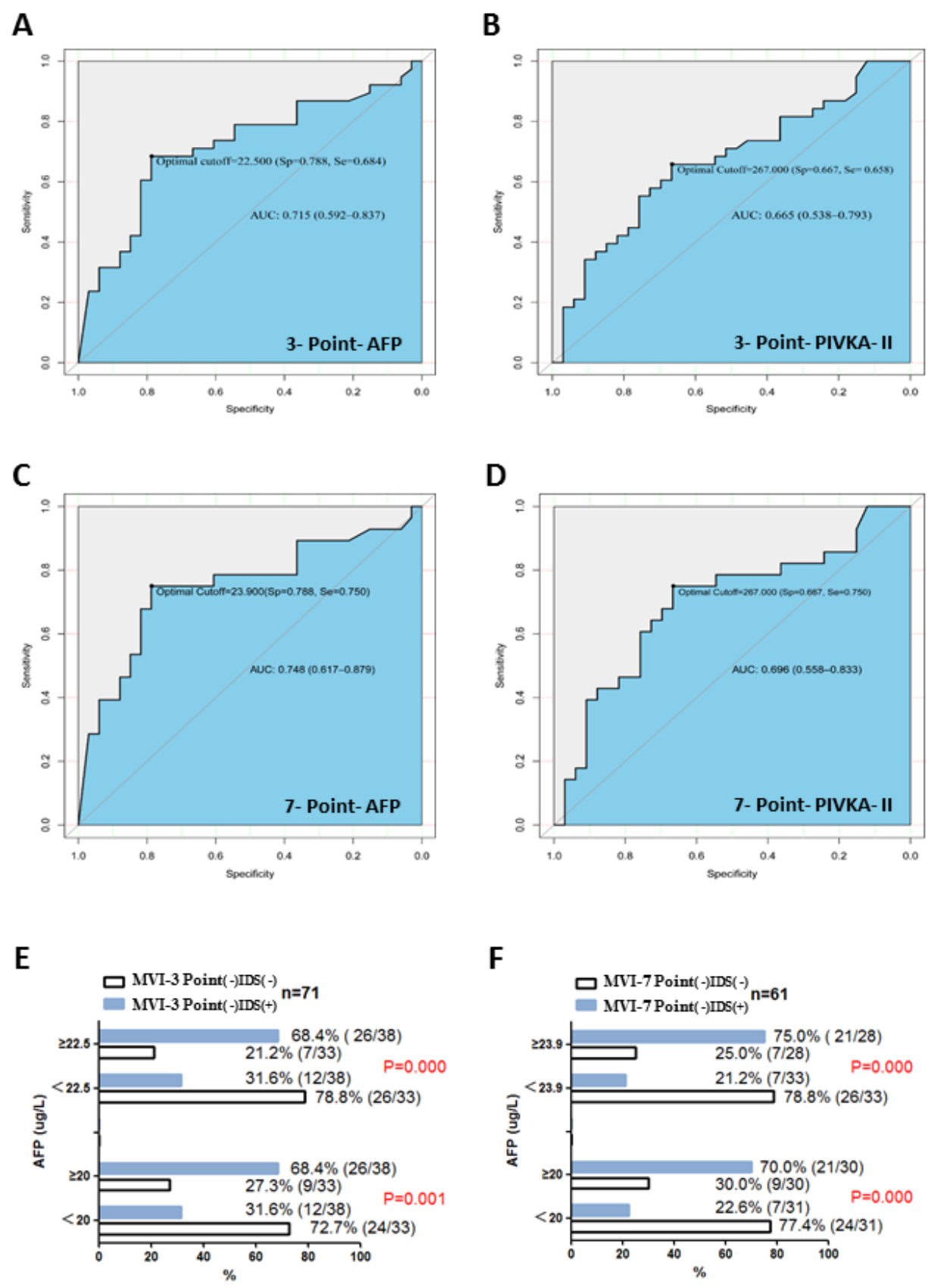

\section{Figure 5}

$A \& B$, The ROC curve of AFP and PIVKA-II to predict MVI false negative status under 3-point baseline sampling protocol. C\&D, The ROC curve of AFP and PIVKA-II to predict MVI false negative status under 7point baseline sampling protocol. E, AFP normal value and cutoff value to distinguish MVI false negative 
status under 3-point baseline sampling protocol. F, AFP normal value and cutoff value to distinguish MVI false negative status under 7-point baseline sampling protocol. 